Addendum: Guidance of regulatory T cell development by Satb1-dependent super-enhancer establishment

\begin{abstract}
Yohko Kitagawa, Naganari Ohkura, Yujiro Kidani, Alexis Vandenbon, Keiji Hirota, Ryoji Kawakami, Keiko Yasuda, Daisuke Motooka, Shota Nakamura, Motonari Kondo, Ichiro Taniuchi, Terumi Kohwi-Shigematsu \& Shimon Sakaguchi

Nat. Immunol. 18, 173-183 (2017); published online 19 December 2016; addendum published after print 17 April 2017
\end{abstract}

ChIP-seq, RNA-seq, MBD-seq and ATAC-seq data sets associated with this article were originally deposited in the DNA Data Bank of Japan under accession numbers DRA003955, DRA004738 and DRA005202. The sequencing data sets have now also been submitted to the NCBI SRA database. The SRA accession number is DRP003376, and all the data (109 samples in total) are accessible at https://www.ncbi.nlm.nih.gov/ sra/?term=DRP003376.

\title{
Corrigendum: NLRP12 attenuates colon inflammation by maintaining colonic microbial diversity and promoting protective commensal bacterial growth
}

Liang Chen, Justin E Wilson, Mark J Koenigsknecht, Wei-Chun Chou, Stephanie A Montgomery, Agnieszka D Truax, W June Brickey, Christopher D Packey, Nitsan Maharshak, Glenn K Matsushima, Scott E Plevy, Vincent B Young, R Balfour Sartor \& Jenny P-Y Ting Nat. Immunol. 18, 541-551 (2017); published 13 March 2017; corrected after print 17 April 2017; corrected after print 18 May 2017

In the version of this article initially published, the vertical axis of Figure $5 \mathrm{j}$ was incorrectly labeled 'PC1 (22.56\%)'. The correct label is 'PC3 (6.47\%)'. The error has been corrected in the HTML and PDF versions of this article.

\section{Corrigendum: The transcriptional coactivator TAZ regulates reciprocal differentiation of $T_{H} 17$ cells and $T_{\text {reg }}$ cells}

Jing Geng, Shujuan Yu, Hao Zhao, Xiufeng Sun, Xun Li, Ping Wang, Xiaolin Xiong, Lixin Hong, Changchuan Xie, Jiahui Gao, Yiran Shi, Jiaqi Peng, Randy L Johnson, Nengming Xiao, Linrong Lu, Jiahuai Han, Dawang Zhou \& Lanfen Chen Nat. Immunol. 18, 800-812 (2017); published online 15 May 2017; corrected after print 20 July 2017

In the version of this article initially published, the description of Figure 1d,e in the first subsection of Results was incorrect; “...(Lck_Cre): Taz ${ }^{\mathrm{fl} / \mathrm{fl}} L c k$-Cre mice) immunized with KLH exhibited a larger $\mathrm{T}_{\mathrm{H}} 17$ population and fewer $\mathrm{T}_{\text {reg }}$ cells than that of their Taz $z^{\mathrm{fl} / \mathrm{fl}}$ littermates..." should read: “...(Lck-Cre)): Taz $z^{\mathrm{fl} / \mathrm{fl}} L c k$-Cre mice immunized with $\mathrm{KLH}$ exhibited a smaller $\mathrm{T}_{\mathrm{H}} 17$ population and more $\mathrm{T}_{\text {reg }}$ cells than that of their $\mathrm{Ta} z^{\mathrm{fl} / \mathrm{fl}}$ littermates.... Also, the second sentence of the panel legend for Figure 1a incorrectly identified the numbers in the plots on the top row as "percent TH7 cells..."; this should read "percent $\mathrm{T}_{\mathrm{H}} 17$ cells..." These errors have been corrected in the PDF and HTML versions of this article.

Corrigendum: YAP antagonizes innate antiviral immunity and is targeted for lysosomal degradation through IKKe-mediated phosphorylation

Shuai Wang, Feng Xie, Feng Chu, Zhengkui Zhang, Bing Yang, Tong Dai, Liang Gao, Lin Wang, Li Ling, Junling Jia, Hans van Dam, Jin Jin, Long Zhang \& Fangfang Zhou

Nat. Immunol. 18, 733-743 (2017); published online 8 May 2017; corrected after print 20 July 2017

In the version of this article initially published, the source of the HSV-1 virus stock used in the study was identified incorrectly as a purchase from the Wuhan Institute of Virology, Chinese Academy of Sciences. The correct Acknowledgements section should begin "We thank F. Zhao (Wuhan Institute of Virology, Chinese Academy of Sciences) for HSV-1" and the second sentence of the third subsection of Online Methods ('Cells and reagents') should read "Herpes simplex virus type 1 (HSV-1) was obtained from F. Zhao (Wuhan Institute of Virology, Chinese Academy of Sciences)." The error has been corrected in the HTML and PDF versions of the article. 